\title{
Final Technical Report on the Genome Sequence DataBase (GSDB): DE-FG03 95 ER 62062
}

\section{September 1997-September 1999}

Special Note: Some of the information provided below was included in the Progress section of the continuation grant which was submitted 4-8-98, however, we don't have confirmation that the information was ever sent as a separate annual progress report, so the information is included here. Also, per Georgia's request I am including a copy of 1997's progress. In addition, the final year (September 1998 through June 1999) covered by this progress report was a nocost extension of the previous year's grant. Consequently development of GSDB tools/capabilities, and data acquisition/curation activities were scaled back significantly.

\section{Overview}

Since September 1997 NCGR has produced two web-based tools for researchers to use to access and analyze data in the Genome Sequence DataBase (GSDB). These tools are: Sequence Viewer, a nucleotide sequence and annotation visualization tool, and MAR-Finder, a tool that predicts, based upon statistical inferences, the location of matrix attachment regions (MARs) within a nucleotide sequence. NCGR added sequence similarity search capabilities in the form of BLAST ${ }^{T M}$ to the suite of tools available with GSDB, with user-friendly features such as the ability to create customized target databases. In addition, to augmenting the tools associated with GSDB, the quality of the data within GSDB was improved by continued, focused data curation. These efforts included the maintanence and updating of the SANBI STACK data set, sequence alignment sets, chromosome information for human sequences, and taxonomic information associated with sequences. NCGR deployed a new database record flatfile format that allowed users to access all of the information associated with sequences in the database. A more detailed description of these tools and efforts is included below.

The final portion of this report details ongoing work to transfer to GenBank the ownership of data which was submitted to GSDB so that continuity of service can be continued through GenBank. It is anticipated that this work will be completed in the early part of the year 2000 . 


\section{DISCLAIMER}

This report was prepared as an account of work sponsored by an agency of the United States Government. Neither the United States Government nor any agency thereof, nor any of their employees, make any warranty, express or implied, or assumes any legal liability or responsibility for the accuracy, completeness, or usefulness of any information, apparatus, product, or process disclosed, or represents that its use would not infringe privately owned rights. Reference herein to any specific commercial product, process, or service by trade name, trademark, manufacturer, or otherwise does not necessarily constitute or imply its endorsement, recommendation, or favoring by the United States Government or any agency thereof. The views and opinions of authors expressed herein do not necessarily state or reflect those of the United States Government or any agency thereof. 


\section{DISCLAIMER}

Portions of this document may be illegible in electronic image products. Images are produced from the best available original document. 


\section{New Tools and Services}

\section{Sequence Viewer}

As a result of user feedback and staff evaluation of our platform-dependent visualization, annotation, and editing tool, 'Annotator', we developed a webbased nucleotide sequence and annotation visualization tool called Sequence Viewer (SV). A major goal in creating SV was to make it less dependent on highend hardware than its predecessor, Annotator, so that more researchers could use it. Other improvements included a customizable graphical display, easier access to data that cannot be represented graphically, and improvements to the graphical display. The SV graphical environment makes it easier for researchers to interpret the biological information associated with a sequence, such as the location of Sequence Tagged Sites (STSs), the type of biological features, and the strand orientation of each denoted biological feature.

Sequence Viewer was implemented in Java and has several modules to allow maximum flexibility and reusability of generated code. The first module that was implemented was the viewer module to provide a graphical view of the sequence and its annotation. The SV was developed as both an applet and as an application. Both versions of SV are freely available at www.ncgr.org/gsdb/sv.

As part of the development of this software, the programming staff developed a new application programming interface (API). The API is software that serves as a middle layer of software between the graphical interface and the data in the database. The use of this middle layer allows client applications like SV to be designed without needing to incorporate all of the details of the database. The previous API matched the GSDB schema so closely that it was difficult to make schema changes without making corresponding changes in the API software. The redesigned API uses more general classes of objects, so that schema changes can be made with minimal changes to the API, and was designed to be compliant with the Common Request Broker Architecture (CORBA).

\section{MAR-Finder}

MAR-Finder is a nucleotide sequence analysis tool developed by Dr. Gautam Singh during his tenure at NCGR. It is used to identify DNA regions involved in attachment to the nuclear matrix. MAR-Finder (www.ncgr.org/MarFinder) is a web-based tool that uses statistical inference to deduce the presence of matrix association regions (MARs) in DNA sequences. MAR-Finder is ideal for researchers interested in DNA structure and identification of genes within a sequence.

The tool has been used to discover a new human gene, SOCS-1. Stephan A. Krawetz, associate professor of obstetrics and gynecology, with the Center for Molecular Medicine and Genetics at Wayne State University used the tool to confirm the presence of a gene that other gene prediction methods had failed to detect. 


\section{BLAST ${ }^{\mathrm{TM}}$ Search Capabilities}

As sequence similarity searching is the primary way in which many scientists utilize nucleotide sequence databases, NCGR used its own resources to purchase a TimeLogic DeCypher system in order to provide these capabilities to researchers. During this time the Blast ${ }^{\top \mathrm{M}}$ family of algorithms was made publicly available.

In support of sequence similarity searching, NCGR developed a program to create a non-redundant nucleotide sequence set. The program generates a nonredundant target set of all GSDB nucleotide sequences in fasta format. This program identifies sequences that have exactly the same length and base composition and includes only one of these in the final target data set. The program is re-run regularly to incorporate new data into the non-redundant data set. After this updating, the data set is formatted for use with BLAST homology searches. By limiting the size of the search space and by limiting the number of matches to essencially identical sequences the analysis speed is improved and the set a researcher has to sort through is simplified.

Another feature in this domain that NCGR implemented was the ability to customize the set of target sequences. This allows researches to limit their search space along specific taxonomic lines and/or to include special data sets. The advantage is that this can reveal relationships that are otherwise eclipsed by matches to sequences in the rest of the database.

\section{Data Acquisition and Curation}

\section{Focused Data Curation}

During this period the staff of GSDB focused curation efforts on specific classes of data including taxonomic information, source chromosome information, and protein coding region boundaries. These three areas were selected because they lay the foundation for data interpretation which researchers utilize in a variety of ways. For example, the specific genetic code or translation table that is used to translate a nucleotide sequence into a protein sequence is determined by the taxonomic information associated with the sequence. If the taxonomic information were inaccurate then the resulting protein sequence might be incorrect. The curation of taxonomic and human chromosomal information was deemed pivotal to NCGR's ability to provide researchers with the ability to customize sequence similarity target sets and in NCGR's ability to maintain the human chromosome representations. These curation efforts included adding new information and updating/correcting existing information. Taxonomic data was curated on a daily basis, while the chromosome and protein coding region curations were executed on a bi-weekly to monthly basis. 


\section{Human Chromosome Representation and Curation}

The previously constructed discontiguous sequence based maps corresponding to all human chromosomes except $Y$ were continually updated and refined based upon information obtained from a variety of web-based resources. These maps included STS markers in the Whitehead Institute for Biomedical Research maps repository and STS markers in the Stanford Human Genome Center repository. In addition, a set of 1400 STS markers specific to chromosome $X$ that were mapped by Washington University at St. Louis were added to the database representation of chromosome $X$. The appropriate web sites were screened on a monthly basis and the corresponding chromosome constructs were updated with the newly gleened information.

\section{Ongoing Efforts}

NCGR is in the process of providing GenBank with the revision histories (primarily the original submission date, original release-to-the-public date, and sequence revision dates) of nucleotide sequences submitted to GSDB so that they may take over the maintenance of these publicly available data records. In addition we will be transferring responsibility of all remain unpublished (i.e. still held in confidence) data to GenBank. In the next month we will notify data submitters of the impending change and provide them with appropriate contact information for future release of data and revisions.

NCGR is continuing to provide access to the data in GSDB via simple query tools and sequence similarity tools. It is anticipated that NCGR will continue to provide these capabilities for at least another year. 


\section{Technical Progress Report}

\section{Overview}

This report summarizes the activities of the Genome Sequence DataBase (GSDB) project from June 1, 1996 to August 1, 1997, which was initiated under the Cooperative Agreement DE-FC0395ER62062 with the Office of Health and Environmental Research in the Department of Energy. The objective of this project is to create and maintain a relational database that supports distributed community sequence generation, annotation, and interpretation of genomic sized DNA sequences. This report supplements the report submitted as part of the July 25, 1996 Continuation Proposal, which described the activities of the first year of the cooperative agreement.

The primary foci of the GSDB project over the last year have been to:

- Utilize the new data representation capabilities of the database.

- Identify areas of data representation that need refinement.

- Design and implement applications to perform database input/output functions, provide web query capabilities, and prototype BLAST/FastA searching capabilities.

- Utilize and disseminate the graphical sequence viewing, editing, and submission tool called Annotator.

- Assess the consistency and quality of data in the database and make systematic improvements where possible.

In November of 1996 the Genome Sequence DataBase (GSDB) completed the arduous task of moving all the data from Sybase version 492 to an improved schema in Sybase version 11. During the past year, the GSDB staff has spent a significant portion of its time and effort focusing on improving the quality of data within GSDB and on improving programs that interact with the database and database services. The quality of data within GSDB has been improved during FY 97 by quality assurance/quality control $(\mathrm{QA} / \mathrm{QC})$ measures being applied to data already existing in GSDB. This QAVC includes the identification of all sequences in the database which contain vector contamination and the verification of database conversion process. Quality has also been improved by the development of procedures/data checks that prevent erroneous data being 
incorporated into GSDB. These include a vector check algorithm. Lastly, the value of the data in GSDB has been augmented by the construction of premier data sets. Examples of such sets are: 1) an alignment set to the protease, glycoprotein and reverse transcriptase genes of the HIV genome, 2) alignments to the complete bacterial genomes, and 3) the sequence based maps for the human genome and other eukaryotic genomes.

In addition, the GSDB programming staff has made improvements in programs and services that are crucial to the functioning of GSDB. These programming projects have ranged from code maintenance and enhancement, to development of new tools for GSDB staff and GSDB users. The following major projects are completed:

- Development and implementation of a web-based query interface (Maestro) and a subsequence retrieval service.

- Redesign and implementation of a suite of programs (Import Suite) that collects data from the International Collaboration (IC) and incorporates it into GSDB. The programs also support manual entry of data into GSDB.

- Development of an algorithm (Merge) and user interface that incorporates sequence/annotation updates from the International Collaboration (IC) into GSDB records.

- Construction of internal BLAST/FastA homology server and publicly available sequence files in FastA format that can be used by BLAST or FastA.

\section{Utilization of New Data Types}

The complete microbial genomes and the complete yeast chromosomes are significant resources to for making sequence comparisons within an organism and between different organisms. The GSDB staff is utilizing this new capability of the database to store sequence alignments on these high. profile sequences. In addition, the staff has been using the new discontiguous sequence data type to create sequence-based marker maps for data from organisms which are the central focus of the research community, e.g., the human chromosomes. 
The goal of this work is to take the publicly available sequence data related to these high profile genomes and organize it into a more logical and useful format. This is achieved by storing sequence relationships in defined data fields in GSDB.

The list of GSDB accession numbers corresponding to the special data sets described below are included in Appendix

\section{Complete Genomes}

Over the course of the last year, there has been a significant increase in major sequencing centers making their sequence and annotation data available via web sites prior to incorporation into one of the public nucleotide sequence databases. In response to this change, the GSDB staff has shifted its focus from merely reviewing data submitted by researchers to actively mining sequence and annotation data that is available via the World Wide Web.

During FY 97, the GSDB staff incorporated the complete genomic sequences and associated biological feature annotation as single sequence records for the microbial genomes that have been completely sequenced. In addition, alignments are being created between these complete genome sequences and other smaller sequences from the same organisms which already exist in the database, including the fragments that GenBank uses to represent the complete genomes. This work is also being performed for the eukaryotic chromosomes which have been sequenced in their entirety. A summary of the complete genomes which have been incorporated into GSDB and the status of process of making alignments to each genome is provided below.

\section{Escherichia coli}

Recently, the sequencing and annotation of the complete Escherichia coli genome was completed. GSDB mined this data in the form of a GenBank flatfile. The sequence record was comprised of 4.6 megabases and had 4334 coding regions, 4346 genes, 1750 miscellaneous features containing 8502 notes, 492 miscellaneous structures, and 14 ribosomal binding sites. The flatfile was reviewed by the GSDB staff and it was determined that approximately 1000 of the miscellaneous features 
were better represented in GSDB as sequence alignments or other more specific features which GenBank is unable to represent. In addition to properly representing the numerous data types that Genbank cannot, GSDB is also generating alignments to the complete genome based on homology to the more than 4700 other $E$. coli sequences currently in GSDB. These alignments will not only facilitate whole genome comparisons, but will aid in elucidating structural and functional motifs within the genome.

\section{Haemophilus influenzae}

The complete genome of Haemophilus influenzae has existed in GSDB as a single sequence, with its nearly 1800 annotated features, for nearly two years. In addition to the complete genome, there are hundreds of smaller $H$. influenzae sequences in GSDB, including pieces which GenBank uses to represent the complete genome. The positions along the complete genome to which these smaller sequences align were determined through the use of BLAST and other algorithms. Based upon this analysis, approximately 1730 sequence alignments have been created to the $H$. influenzae complete genome and they are contained within GSDB.

\section{Human Immunodeficiency Virus (HIV)}

The identification and functional analysis of mutations within the HIV genome are of current medical interest because of the high mutation rate of this virus. To date, portions of the small genome have been sequenced from hundreds of different patient isolates and stored in GSDB. In order to increase the use of these sequences, the GSDB staff has constructed alignments to the complete sequence of the reference strain of HIV 1 (strain HXB 2) for the pol (reverse transcriptase, protease, integration protein) and env ( 2 envelope glycoproteins) genes. Alignments were constructed for the majority of public sequences for these two genes. Approximately 65 alignments were constructed for pol sequences and 1800 for env sequences.

\section{Methanococcus janaschii}


Both the bacterial chromosome and the two extra chromosomal elements which comprise the complete genome of Methanococcus janaschii are contained within GSDB (each as a single sequence). The GSDB staff created approximately 150 alignments between the chromosomal sequence and other smaller $M$. janaschii sequences in GSDB, including the pieces of sequence GenBank uses to represent the chromosome. Similar alignments are being created for both of the extra chromosomal element sequences.

\section{Mycoplasma genitalium}

As with $M$. janaschii and $H$. influenzae, Mycoplasma genitalium exists as both a single sequence and as individual pieces created by Genbank. We are in the process of creating alignments between the Genbank pieces and the complete sequence for $M$. genitalium. Once these alignments are complete we will add alignments for other $M$. genitalium sequences that are in the database.

\section{Rhizobium sp. symbiotic replicon pNGR234a}

Earlier this year, an agronomically important extra-chromosomal plasmid, the Rhizobium sp. symbiotic replicon pNGR234a, was completely sequenced and annotated. The agronomic importance of this extra-chromosomal plasmid lies in the fact that it appears to code for many of the enzymes involved in fixation of atmospheric nitrogen. In July 1997, the GSDB staff imported the complete $536 \mathrm{~kb}$ sequence into GSDB. We are currently in the process of generating alignments based on homology to all sequences from species of Rhizobium, Bradyrhizobium, Sinorhizobium, and Agrobacterium. There are more than 900 sequences from these 4 genera in GSDB, which could share homology with NGR234. Initial BLAST analysis identified only 7 sequences with high homology to NCR234. Alignments between these 7 sequences and the complete plasmid sequence have been created. Further analysis is underway to determine whether any of the other 890 sequences contain significant homology to this plasmid.

\section{Saccharomyces cerevisae}


The complete genome of Saccharomyces cerevisae, which is comprised of sixteen chromosomes, has been fully sequenced. The Yeast Genome Project at Stanford University has completed the annotation of 5 of these chromosomes based upon their structure-function analysis. In May 1997 the GSDB staff mined all of these sequences from the Stanford site, began entering them into GSDB, and generating alignments. There are approximately 10,000 S. cerevisae sequences in the world's databases; these form the base sequence population from which alignments are being generated. BLASTn 1.4.9, BLASTn 2.0 and FastA are being used serially to determined homology. We completed work on chromosome VIII in June 1997, and have generated over 80 alignments to that sequence. In addition, chromosomes III, VI, and $\mathrm{XX}$ are now incorporated into GSDB and approximately 115,110 , and 160 respective alignments have been created. The importance of alignments cannot be overstressed in that they allow cross-sequence comparisons at both the sequence and the feature levels, thereby minimizing duplicity of effort in functional analysis. They also provide a means of double checking analysis results. We are presently focusing efforts on chromosome $\mathrm{V}$, the last chromosome with relatively complete annotation, and may begin to perform structure/function analysis on the other eleven chromosomes independent of the Yeast Genome Project.

\section{Synechocystis sp.}

Following the announcement of the completion of the blue-green algae genome, Synechocystis sp., the GSDB staff began monitoring the Kazusa DNA Research Institute web site. In March 1997, when the complete sequence and its feature annotation became available GSDB staff downloaded the data from the Cyanobase web pages. Using internal software, GSDB staff converted the tabdelimited annotation data into GIO format for insertion into the database. After conversion into GIO, staff reviewed the data using Annotator, checking for accurate annotation. Once the quality of the data was verified, the sequence and annotation were inserted into the actual database and released to the public.

\section{"Bugs-n-Stuff" Information Web Pages}


Initially, when the first few complete bacterial genomes and their annotations were submitted to GSDB, the GSDB staff prepared informational web pages about each specific organism. These information pages were made publicly available at the same time as the complete bacterial genome record (such as H.influenzae and M.genitalium). These information pages about microorganisms were very well received by the research and educational communities. As a result of this interest, efforts to provide information about the microbial genomes which are being sequenced (in the public sector) has expanded into web pages called "Bugs-n-Stuff". These microbial genome pages were created as a reference for the community and contain a list of current or completed eubacterial, archaeal and eukaryotic genome sequencing projects. Each main page includes the name of the organism being sequenced, which sequencing group(s) are involved in the effort, background information on the organism, and its current location on the Carl Woese Tree of Life. As the Microbial Genome Initiative progresses, this project will be updated on a periodic basis.

\section{Discontiguous Sequences and Sequence Based Maps}

Improvement of data utility has been a major focus during the past year for the GSDB staff. One focal point of this effort to improve the utility of the data involves organizing sequences into logical groupings, which are referred to as discontiguous sequences. The scale of a discontiguous sequence can range from as small as the exons which comprise a gene to as large as an entire chromosome. The GSDB staff constructed discontiguous sequences to represent chromosomes of the human, maize and rice genomes. Following the construction of the basic chromosomal representation, which primarily included STS markers, large genomic sequences and other "high" interest sequences, these data were compared at the sequence level to the components of each discontiguous sequence. This protocol served to identify other sequences which should be incorporated into the chromosomal representations. Many standardized protocols for data manipulation were established during the construction of these chromosomal representations which will be useful in the future.

\section{Human Sequence-based Maps}


The GSDB staff has constructed discontiguous sequences for human chromosomes 1 through 22 and $X$ that include the STS markers from the Whitehead Institute for Biomedical Research maps and STS markers mapped by the Stanford Human Genome Center. The set of 2000 STS markers for chromosome $\mathrm{X}$ that were recently mapped by Washington University at St. Louis have also been added to chromosome X. Approximately 50 genomic sequences have been added to the map of chromosome 22 by determining their overlap with the mapped STS markers. Genomic sequences are being added to all of the chromosomes as their overlap with the STS markers is determined. These discontiguous sequences can be easily retrieved and viewed via their sequence names using the GSDB Annotator. The sequence names follow the format of: HUMCHR $\# M M$, where \# equals 1-22 or $\mathrm{X}$.

\section{Stack Sequences}

In May, 1996, Winston Hide of the South African National Bioinformatics Institute (SANBI) visited GSDB to discuss the submission and processing of the Sequence Tag Alignment and Consensus Knowledgebase (STACK) through GSDB. Since Dr. Hide's visit, we have maintained daily communication with the SANBI genome center, assisting the center with creating syntactically correct GIO files, reviewing their sequences and providing them with the means to review, and processing the sequences into the database.

STACK is a large number of CDNA sequences processed and assembled with the aim of generating high quality consensus sequences. STACK may be the largest set of coding human genome data ever assembled. Ultimately, the entire collection will be submitted and made publicly available. SANBI has already sent GSDB approximately 4000 consensus sequences, which are currently being reviewed.

The GSDB staff is currently working with the Dr Hide to incorporate these sequences into GSDB and the staff is working to align these sequences to the human chromosomal discontiguous sequences. 


\section{Related Agricultural Data Activities}

Most of the efforts of the GSDB staff are supported by the cooperative agreement with the Department of Energy; therefore most of the database activities are focused around the human genome, microbial genomes and other model organism genomes. However, in January of 1997, GSDB's ability to represent sequence-based chromosomal maps and sequence alignments was demonstrated at the Plant and Animal Genome Meeting in San Diego. As a result of interest generated at this meeting, some funds through a cooperative grant with New Mexico State University (NMSU) have been received and are being used to support efforts to construct sequencebased maps of some agronomically important crops. The construction of a maize map and a rice map are discussed below. Collaborations in research areas like agriculture are very beneficial to GSDB because they not only increase the database's potential funding sources, but also increase the database's user community.

\section{Maize Sequence-based Maps}

Chromosomal map data obtained from the Maize Genome DataBase (Maize DB) were used to construct discontiguous sequences corresponding to the UMC 96 maize chromosome maps. The identity and positional information of the sequences which were included in the discontiguous sequences were verified by cross checking with the original data in Maize DB. In addition, protocols to convert centiMorgan $(\mathrm{cM})$ values to kilobases and to represent markers that presently do not contain sequence data were established for the maize maps.

The maintenance of these discontiguous sequences, requires the periodic "mining" of map data from Maize DB. Several scripts were written to streamline this process. These scripts will serve as models for the automation of data mining from other databases and web sites. In addition to the UMC 96 maize chromosome maps there are two other agronomically important strains of maize for which maps exist. Future work will not only include the maintenance of the UMC 96 map, but also the creation and maintenance of discontiguous sequences representing the Brookhaven National Laboratory and the USDA-ARS maize maps. Funding for this work will continue to come from the cooperative grant with NMSU. 


\section{Rice Sequence-based Maps}

In February and March, 1997, the GSDB staff built twelve discontiguous sequences, with each representing a chromosome of Oryza sativa v. kasalath $x$ nipponbare, and encompassing all available mapped and sequenced elements from the RiceGenes and Japanese Rice Genome Project databases. Positional data for markers were obtained from traditional genetic maps. This required the conversion of $\mathrm{cM}$ values to kilobases based on the overall physical size of each chromosome divided by the total number of $\mathrm{cM}$ per chromosome. As map markers are added, the kilobase values will be updated to reflect the changing genetic distances. Changes in the GSDB schema are planned to allow the inclusion of sequenceless data, which will facilitate the representation of genetic markers and quantitative-trait loci (QTL). At such time, GSDB will be able to represent the complete genetic and physical maps and update these maps as more markers are sequenced, with the ultimate goal of generating complete contigs of all twelve chromosomes, fully indexed and cross-referenced by loci. Such contigs will prove invaluable in whole genome comparisons of grass species. Future work on the rice map will continue to be supported by the cooperative grant with NMSU.

\section{Data Representation Refinements}

The ability to support new data types such as discontiguous sequences, sequence alignments, and sequences of unlimited size is of great value to GSDB and the research community. However, the use of these new data types by the GSDB staff has demonstrated that a few improvements are still needed. Many of these improvements involve schema changes.

\section{Schema Changes to Support Genetic Distances}

In the construction of the human, maize and rice chromosome representations it became clear that to accurately represent some of these sequence-based maps GSDB needs to be able to incorporate true genetic map information. The appropriate schema changes to support centiMorgan and centiRay map information associated with the sequence pieces of discontiguous maps were 
designed, reviewed, approved, and implemented. The support of these data types will aid researchers by allowing them to represent both genetic and physical distances for a sequence within a map so that correlations can be made between two types of measurements.

Additionally, during the construction of the various chromosome map representations, we discovered that many of the markers localized to specific regions of a chromosome have not been sequenced. These markers may be RFLPs or QTLs, or may have been sequenced in another organism and only hybridized to the chromosome (in the organism for) which we are constructing a discontiguous sequence. This necessitated additional changes to the schema to support accession numbers that do not contain any basepairs. The requirements for this set of improvements has been reviewed, approved and implemented. This will eliminate the need to put "place-holder" sequences, such as the restriction enzyme recognition sequence, into the database to place these markers in the discontiguous sequences.

\section{PROGRAMMATIC IMPROVEMENTS}

During the past year the GSDB Information Technology (IT) group has completed a variety of projects. These projects have ranged from code maintenance and enhancement to development of new tools for GSDB staff and GSDB users. The major projects that have been completed are:

- Development of Maestro query interface and a subsequence retrieval script.

- Reengineering of the Import Suite of programs.

- Development of Merge algorithm and user interface .

- Construction of internal BLAST/FastA server and publicly available BLASTable sequence files.

In addition to these major projects both sub-sections of the IT group (Web development and Software development) have completed numerous other tasks that support both internal staff and external user needs. 
Provided below is a detailed description of the major programmatic projects. In addition the minor tasks of the IT group will be explained relative to the Software and Web group sub-sections.

\section{Maestro}

Since the web is a common interface for many tools, and web based applications are cross platform, we have implemented a development plan for implementing web based tools. Our effort has focused on developing tools which are intuitive for the user and allow discreet access to the information contained in GSDB. An improved query interface (Maestro) has been developed pursuant to the conversion of the GSDB schema and to meet the needs of the biology community.

The first phase of this development plan is GSDB Maestro. With Maestro, users can query a variety of database fields, simply by providing a valid searchable term. Logical operators can also be used to create compound queries, which further refines the extraction of desired data. All queries can output data in GSDB flatfile, FastA, and GIO formats.

During the past year the GSDB IT group has developed Maestro from concept to production. The development process has included the creation of a $\mathrm{C}+$ program that interacts with a specialized Maestro database tables within GSDB. The Maestro database tables were created as a unique subset of the complete GSDB. With this specialized set of database tables the querying time for complex GSDB queries was reduced from 3 minutes to 3 seconds. In addition, a process has been developed to update the tables in the Maestro database tables with new information which is deposited into GSDB. This update process consists of a tiered system of database table construction in conjunction with optimization. Currently the Maestro database tables are being updated on a weekly basis.

A graphical user interface (GUI) was developed by the web group to allow easy navigation and uniform look and feel. This GUT interacts with the suite of programs/scripts that were written to access, collate, and present information generated by Maestro queries. 
Maestro supports the querying of 18 different database fields based upon user entry of an associated valid term. A user can retrieve sequence documents by simply entering a search term, and selecting a database field from a pull-down menu. In normal mode, Maestro will retrieve all exact matches of the user's query, and return an indexed list of similar terms if no exact match is found. In wildcard mode, Maestro will return a list of all terms matching the wildcard query criteria. An additional function that was added is the sequence length filter. Once a match has been obtained for a given query you can refine the query by specifying a range of sequence lengths. Using the sequence length filter, a user can indicate the minimum, maximum, or range of sequence lengths to be returned, for sequences matching the given query criteria.

In short Maestro is our first step in creating reliable, usable, and intuitive tools for accessing information in GSDB via the World Wide Web.

\section{Subsequence Retrieval Script}

The sequence records of complete genomes are often extremely large and many users have either software or hardware that restricts their ability to utilize these large files. In addition, many researchers are only interested a segment of the complete sequence. To address this problem, GSDB staff wrote a program that offers four options for retrieving a selected segment of any accessioned, public sequence. The user may select a simple base pair span, any span with a given gene or product name, all genes, or all intergenic regions. This capability is currently being tested and will be available on the GSDB web page by the end of 1997.

\section{Import}

The Import Suite is a series of programs that collect sequence entries from either the ftp sites of the IC databases (via cron-scheduled jobs that run nightly) or user-supplied files (via the Manuallmport application), and store this information in GSDB. In the former case, these entries are in the ICdefined "flatfile" representation of sequence data, while in the latter case they may be either flatfiles or the special GIO file format that was designed as a file-based representation of the GSDB schema. Import is thus primarily responsible for mapping the information in these files into the 
GSDB schema. If GSDB already contains a version of an entry, Import will attempt to reconcile any differences that exist between the foreign representation of the entry and the GSDB version via the Merge algorithm. Import interacts heavily with the Tracker database in order to leave a record of its actions on GSDB, including a log of the errors that it encounters.

Among the improvements to the Import suite that have been completed in the past year are the following:

- Flatfile parsers provide better mapping to the GSDB schema.

- Flatfile parsers have been "modularized", meaning that the import of an entry is no longer dependent on the entire flatfile being parsable in terms of the grammar used in the mapping; subsections of a flatfile can fail to parse, and will be flagged for review, while the rest of the information will be successfully imported into GSDB.

- Processing of nightly updates from the IC databases is no longer dependent on consistent distribution of segmented sets; Import can correctly handle the commonly seen case where only a subset of the entries of a set are included in the distribution file.

- Import now correctly and automatically processes complete releases from the IC databases, in order to obtain any changes to their entries that they have not been included in the nightly update distributions.

- An improved version of Merge (2.0) has been incorporated into Import, to provide better tracking for insertion and deletion of features.

- Cron-scheduled processes have been streamlined to perform Import nightly on the data from the IC databases with a minimum of manual intervention required (for error handling); reports are generated nightly to inform staff of the status of these processes. 
- A utility for in-house use, Manuallmport, has been developed for import of entries obtained from other sources (direct submission, data mining efforts).

- Utilities for reviewing and correcting entries that fail to import have been developed for inhouse use.

\section{Merge Project}

The objective of the Merge project was to take the existing information in a flatfile (which is stored in GSDB) and "merge" it with an update of the same flatfile record from one of the IC databases (DDBJ, EMBL, GenBank). An algorithm was developed by the IT staff to achieve this task. Updates from the IC databases are distributed once a day, consequently GSDB executes the Merge algorithm daily.

Updates to flatfiles may involve changes to the sequence, changes to feature annotation or both. The GSDB data network merge operation underlies the higher level need for the ability to intelligently import features from the updated flatfiles of the sequences existing in GSDB. The annotations (including features) on the resulting GSDB entry will either be retained, modified or deleted.

The primary function of the Merge software is to perform an intelligent merging of IC updates to avoid the assignment of "new accession numbers" to features whenever possible. This task is accomplished by translating the coordinates of features on the old entry to that of the new entry's coordinate system. After the coordinate justification has been performed, the task of matching old features to the new ones is considerably simplified.

In addition to the creation of the Merge algorithm, a graphical user interface (GUI) was constructed to allow GSDB staff to view merged entries for accuracy and then to update the merged entry status in the tracking database and GSDB. The initial state of the merged entry is pending. This designation denotes that the merged entry has not been perused by one of the GSDB curation staff. The interface then allows the GSDB staff member to set the status of the merged entry to "success" 
or "failure". If a merged entry receives a failed designation the entry is then set back into the queue for merging. If the status is "success", then the merged entry is set in the queue for the Import Suite to input into GSDB.

\section{BLAST/FastA Service}

The internal homology search web interface is a prototype used both for algorithm testing and research. In order to accomplish this, generic compilation scripts were written for both the BLAST and the Fast A type algorithms. These scripts allow versions of the algorithms to be easily updated. The interface to the homology search algorithms consists of an HTML form which requests user name, user e-mail address, homology search algorithm type, reference database, and a query sequence in FastA format. The interface also contains links to descriptions of the reference libraries, homology search programs, and the usage of the individual algorithms. A CGI program runs the homology search based upon these input parameters. This same program then reformats the output of the search into a hyperlinked list of results.

The FastA output replaces the difficult to read ASCI histogram with a print quality bar-chart. The creation of this image occurs in two phases. The first is the creation of the postscript version of the histogram using a plotting program that can accept scripted commands. Towards this end we used plotxy, a versatile plotting program written by Robert Parker and Loren Shure for the Institute of Geophysics and Planetary Physics at the University of California San Diego. This postscript image is then translated into a GIF image using the publicly available ghostscript and pbmplus programs.

Additionally, to augment the BLAST/FASTA tool, a preliminary system for adding the results of homology searches to the database was developed. This development entailed a program that converts BLAST output and paracel Smith Waterman (SW) output to GSDB Input/Output File (GIO) update file and an additional program that converts FASTA output and paracel SW output to GIO update file.

\section{BLASTable Sequence Sets}


GSDB provides the research community with some unique sequence sets that researchers are interested in utilizing and that are not currently available through the IC databases. However, the utility of these unique sequences to the research community is limited by the lack of homology search capabilities against sequences in GSDB. Such search capabilities are not possible until an adequate homology search server has been purchased. In the interim, GSDB has created FastA formatted sequence files which are automatically updated on a weekly basis. These files can be obtained from the NCGR ftp site and used by researchers to perform homology searches of the sequences locally on their machine. Currently, the sequences in the database are divided into 14 files based upon taxonomic information associated with each sequence. For instance, there is one file that contains only human sequences and another that contains all mammals except humans. In addition there is a file that contains all of the CDNA and EST sequences in the database. The GSDB staff anticipates adding more FastA formatted files to this set which will be defined by other parameters, for example there all of the sequences that are part of the human discontiguous sequences will be made available at this site.

The construction of these files allows us to provide researchers with the ability to download sets of sequences and conduct homology searches against GSDB data on their local machines. These files can also be used as the search sets for a GSDB search server when it is obtained. In addition, it is clear that some researchers prefer running homology searches on their local machines, therefore even after GSDB has a homology search server, GSDB will continue to provide these data files from the ftp site.

\section{Additional Web Activities}

In addition to Maestro the Web group has been involved in the following activities to improve the GSDB web site:

- Revamped the look and feel. This process included new graphics, better navigation, and the addition of relevant HTML pages.

- Added user account registration for GSDB site. This sub-section of the GSDB site was ostensibly added as a registration page for receiving a beta copy of the GSDB Annotator. 
Additionally the account registration page is being used for new user accounts for SQL access to GSDB.

- Updated the releases of the GSDB Annotator for downloading from web.

- Translated all GSDB documents (e.g. Schema, Annotator User Manual) into Portable Document Format (PDF).

- Updated the Tracking DB scripts to reflect changes in the Tracking DB schema. Also made changes and added functionality in accordance with the needs of the GSDB staff.

- Wrote scripts and modified a statistics programs to tabulate the usage of the GSDB site. Currently the statistics are generated nightly and archived on a monthly basis for internal review and utilization.

\section{User and Software Support}

GSDB adheres to the philosophy that the database is a "community-owned" resource that should be simple to update to reflect new discoveries about sequences. A corollary to this is GSDB's conviction that researchers know their areas of expertise much better than a database curator and, therefore, they should be given ownership and control over the data they submit to the database. Beginning in September 1996, the GSDB staff implemented a program that allows researchers to obtain a password protected database account. This account can be used by researchers to submit, edit, and/or update their own data. In addition, this account can be used to add annotation to sequences that were submitted to the database by other researchers (which is referred to as community annotation). To simplify the process of obtaining a GSDB account, the staff has implemented a web-based request form. The GSDB staff generally processes these requests within 24 hours from the time of receipt.

Researchers with GSDB accounts access the database primarily through the use of Annotator which is a graphical database interface. This software provides researchers with the functionality to view, submit, edit, and update sequences in GSDB. The first beta-test version of Annotator was released to the public in October 1996. Many improvements have been made since then and currently the third beta-test version is available from the GSDB web site for Solaris/Unix and MacIntosh Power Book platforms. A windows NT version is currently under development. 
Annotator is unique among database issued software because it allows direct account controlled access to GSDB. By using Annotator a researcher can graphically access all of the data types that are a part of GSDB. Annotator can also be used to submit, edit and update data within GSDB.

The GSDB staff provides both telephone and e-mail support to researchers who need assistance to obtain an account, use Annotator, and retrieve data from the database. This support ranges from guiding users through entering new data to providing information about the software to assisting in the retrieval of data. All queries and requests are handled in a timely fashion.

\section{DATA Quality and Consistency Improvements}

During the past year the GSDB staff focused on two major projects to improve the quality of data in GSDB. One project involves the examination of the data that was migrated from the previous to the current GSDB database to ensure that data consistency was maintained during the process. The other project involves the identification and subsequent removal of vector contamination in sequences. These two projects are described in detail below.

\section{QC of Data Following Database Conversion}

After conversion to the new GSDB 1.0 database was completed last year the GSDB staff began a systematic comparison of a subset of the migrated data to ensure that data integrity was maintained. Several PERL programs were written expressly for this purpose and at this time quality assurance testing has been completed on a representative subset of the data contained within the Sequence and Source tables. This testing identified several situations/instances where data were duplicated (appeared as two separate entries in the database) in an inappropriate manner. All instances of these errors were identified and systematically corrected in the database. In addition, it was determined that the creation date that is associated with each sequence in the database was not properly migrated from the old database to the new GSDB. The GSDB staff is in the process of implementing a script that will automatically extract the creation date for each sequence from the old database and associate it with the same sequence in the new GSDB. The systematic 
examination of the data that was migrated from the old database to the new GSDB will continue until all data fields have been examined and all inconsistencies have been corrected. Once this examination has been completed the old database will be permanently retired.

\section{Vector Identification and Removal}

The GSDB staff has systematically examined the issue of vector contamination of sequences in GSDB. Given that most vector contamination is likely to arise from DNA sequencing methodologies, the investigation focused primarily on possible contamination located at either the $3^{\prime}, 5^{\prime}$ or both ends of each sequence. The vector sequence that is adjacent to a cloned sequence is usually a multiple cloning sequence (mcs) or a polylinker. The approach that was used to identify potential vector contamination was to create a database of mcs sequences and evaluate sequence homology between each sequence in GSDB with the mcs sequences. At present there are an estimated 2500 unique cloning vectors that are commonly used in molecular biology laboratories. The mcs sequence database contains a unique set of 180 different vector mcs sequences that are estimated to represent the majority of plasmid, phage, and cosmid-based vectors that are routinely used in the laboratory.

During the examination of approximately 850,000 sequences it was discovered that throughout the database, less than $1 \%$ of the sequences contained vector contamination (total of 3029 contaminated sequences). Out of these sequences, $80 \%$ or 2400 sequences contained a restriction enzyme site adjacent to the vector sequence. Over half of these restriction sites were for either EcoRI or BamHI , which are two restriction enzymes commonly used in molecular cloning. These findings were taken as further corroboration that the portions of the sequences that were identified as vector contamination were truly vector sequences.

Further analysis of the vector contaminated sequences suggest that the number of contaminated sequences that are incorporated into database has been decreasing since 1994. This may lead one to believe that the problem of vector contamination is no longer a significant issue for the public nucleotide sequence databases, however, approximately $75 \%$ of the vector contaminated sequences that were submitted to the database in 1997 were EST sequences. Given how frequently ESTs are 
searched for homology, the inclusion of vector contamination in these sequences can create very misleading search results which adversely affect the direction of research that is followed.

The GSDB staff is in the process of developing a system to remove the identified vector contamination from the sequence and to include the vector sequence with appropriate annotation in a comment attached to the sequence. By doing this, GSDB will prevent the vector sequence from influencing homology search results while preserving the original data.

\section{DataBase Facts and Statistics}

At the current rate of data acquisition, the amount of sequence data in GSDB doubles approximately every 14 months. In the past year, GSDB has grown in size from 13 gigabytes to 29 gigabytes. There are currently $1,637,147$ public sequences in GSDB. Of these approximately $58 \%$ are from humans, $5 \%$ are microbial sequences, $3 \%$ are from plants, $2 \%$ are from rodents and nonhuman primates, and $33 \%$ are from other organisms (Figure 1). Similarly, there are 1,076,481,102 total base pairs in GSDB, with $46 \%$ from humans, $10 \%$ from microbes, $7 \%$ from plants, $4 \%$ from rodents and non-human primates, and 32\% from other organisms (Figure 2). Since January 1997, 493,767 sequences have been added to GSDB, representing 326,094,564 new base pairs of sequence.

The database and the Sybase server have evolved into a fully functional, constantly monitored, 7 day $\times 24$ hour, with nightly backups to disk and tape. In addition, the transaction logs are backed up on an hourly basis. If the database becomes unusable due to hardware/software failure, the maximum amount of data loss is 1 hour. If a problem occurs involving the devices where the backups are stored, the maximum amount of data loss is 24 hours. In the past year, the database has been reloaded from backups 3 times due to hardware/software failures or redesign and each time little or no data/database work was lost.

The Sybase auditing system was installed on the server. This provides important information on the number and type of operations performed on the server and database. It also allows for capacity planning and growth charting. Auditing also provides the computer staff with data on who was 
accessing the server/database at any given time. Lastly, the use of database auditing assists the GSDB database administrator to identify and rectify situations that can lead to database downtime before serious problems occur.

In addition to the GSDB production database, NCGR also maintains a test database of similar size, and identical schema. This has been constructed for use by both GSDB's biological and computer staffs for the purpose of testing new data, programs and scripts. This database is also maintained in a 7 day $\times 24$ hour state.

Many scripts have been written to automate various day-to-day operations, for example, the script "dump_gsdb.rds" (code is included in the appendix) is executed on a nightly basis. This script backs up the production database, verifies the success of the backup, compresses the backup and archives it onto a tape. Then it updates files that track the history of the database growth, removes the previous day's database log and transaction log backups. Because of scripts like "dump_gsdb.rds" little manual intervention is now required for the database to run smoothly. In addition, minor changes to the schema have been implemented to improve performance, and to accommodate applications developed by the computer staff.

\section{FUTURE DIRECTIONS}

In the last year the primary focus of GSDB has shifted from the daily processing and review of sequence submissions from individual researchers and genome centers. Sequence submissions are still being accepted from researchers who understand that GSDB is not a member of the International Collaboration and that data submitted to GSDB, at this time, will not appear in the International Collaboration databases. In general, the new focus of GSDB is to:

- Make the data in GSDB more accessible to researchers.

- Actively incorporate data into the database that would not otherwise appear in a public nucleotide database. This includes data mining, construction of high profile sequence records, and establishment of collaborations with external researchers.

- Increase the value of data in the database by identifying/correcting erroneous data and prevent the addition of new erroneous data to the database when possible. 
The GSDB staff has designed the following 10 projects for the next year. These projects will serve to increase the value and usefulness of the data in GSDB.

\section{GSDB flatfile}

In the summer of 1996 GSDB underwent a major database conversion. Many new data types were added to the database. Currently these new data types can only be accessed through the use of a graphical interface, Annotator. The GSDB staff is aware that this method of data access is not convenient for all users and that many database users would prefer to view the data in a flatfile format. The staff has reviewed the GenBank flatfile format and it has designed representation for the new data types which follows the patterns established by the GenBank flatfile. In addition to all of the data types represented in the GenBank flatfile, the new GSDB flatfile will represent sequence confidence values, sequence alignments, user defined features, discontiguous sequences, external database links, and analysis data. The implementation of this new GSDB flatfile is expected to occur by December of 1997 .

\section{GSDB Web-based Data Viewer}

After further evaluation of the Annotator we have concluded that a web based Java applications needed by the scientific community. The major limitations of the Annotator are platform dependency and necessity of a large allocation of computer resources by the end user. In accordance with this analysis we have started the process of writing requirements, specifications, and design documents for a web based Java sequence viewer. In the initial stage of design we have decided that this viewer needs to be constructed in a modular structure to allow maximum flexibility and reusability of generated code. For example if a user desired to submit or edit data they would only need to use the edit module. Similarly if a user desired to only view and query the data they would only need the viewing module and not the editing module. This modular structure allows the user to define their needs relative to their computer resources. Additionally this modularity will be incorporated into all developed applications while attempting to be compliant with CORBA (Common Object Request Broker Architecture) specifications. This 
reallocation of resources to a web based interfaces is necessary to utilize the strengths of the current staff and to meet the needs of the genomic community.

\section{Data Mining}

Efforts to identify novel data sets that can be obtained from web sites or private researchers will be increased over the next year. Many of the DOE and NIH supported genome center web sites will be scanned on a regular basis to mine data that is not supported by the other public nucleotide sequence databases. These data will be incorporated into GSDB and associated with the appropriate sequences. GSDB will mine sequence data from the web sites of other institutes where the data has not been submitted to one of the International Collaboration databases. Appropriate credit will be given to the institute from which data is mined and when possible links will be made back to those web sites.

By proactively incorporating both unique sequences and associated data, GSDB will continue to distinguish itself from the other public nucleotide sequence databases.

\section{Premier Data Sets}

The complete sequence of the $E$. coli genome with 2500 other $E$. coli sequences aligned and 4500 annotated genes is an excellent example of how the new data types in GSDB can be used to showcase a sequence and to best represent all of the publicly available data from a sequence. The GSDB staff is committed to continuing this type of data analysis and processing for all of the complete microbial genomes and complete chromosomes from other model organisms as they become publicly available. The discontiguous sequences of the human chromosome maps (described previously) are another excellent example of how the new data types allow more complete information to be associated with the sequences in the database. Work to maintain the existing discontiguous sequence maps (human, corn, and rice) will continue and similar maps will be made for other organisms when appropriate.

\section{Species Specific BLAST Report}


Each new sequence which is incorporated into GSDB will have a homology search performed against all of the sequences in the database from that organism. Matches greater than a specific threshold will be reported to the staff. When appropriate sequence alignment sets will be created to represent the relationship between newly incorporated sequences and sequences previously residing in GSDB and sequences will be added to the discontiguous maps. This work is dependent upon NCGR obtaining funds to purchase a homology search server.

\section{Collaborations}

We are beginning collaboration with the US Army Missile Command (MICOM)and the Medical Research and Materiel Command. We are working with D. Halitsky and J. Fresco who are developing a database for use in pathogen identification and characterization. As part of this work, they will be identifying codon bias in short sequences and how it relates to structural motifs. The organization of the database will reflect the evolutionary principles of codon usage. Their conception of codon bias and classification of codons is very different from the traditional treatments of these subjects. We will be developing changes to the current GSDB schema to allow the incorporation of data on subsequence length, codon bias, and ratios of codon usage.

\section{Product tree}

Searching the database for sets of sequences, such as all of sequences which contain "botulism related toxins", is not a simple task. Currently the database is designed to query on product names or keywords which all contain the same character string like "botulism", but as in the example of botulism related toxins, many of these toxins have specific names which do not include the word "botulism". The GSDB staff has been examining ways to solve this problem. We are currently testing the idea of using a Product/Keyword tree structure to allow for this type of querying. Two approaches are being tested: one is the use of a hierarchical database structure and the other is the use of an indexed/linked table. The nodes within this structure will be curated and reviewed by both our staff and experts in various areas of protein research. 
Once this system been perfected it will also be implemented to support the taxonomic data in GSDB. This change in structure will make it much easier to query any node in the taxonomic tree and it will make it easier to review and curate taxonomic data.

\section{Synonym table}

Many genes and gene products have been known by different names over time and by different research groups. Currently in GSDB, only the current "official" gene and gene product names are stored in the gene and product tables. This can lead to frustration on the part of a researcher who searches the database using an alternative name. To remedy this situation GSDB is creating synonym tables that will associate all alternative gene or product names with the appropriate official name. The synonym tables for gene and product are currently under development. They are likely to be structured as look-up tables. The synonym table for product will be implemented first, followed by the one for gene names. Upon completion of these two synonym tables GSDB may also design and implement a synonym table to manage all of the common names associated with the scientific names of organisms.

\section{Data Checkers and Reports}

GSDB has a strong commitment to increasing the quality of data within the database. One approach to preventing bad data from being incorporated into GSDB is the use of data checkers and data reports. In the next year GSDB plans to increase the number of biologically relevant data checkers that examine data as it is deposited in GSDB. The rules for the data checkers will be determined by the GSDB biology staff and will be implemented by the database administrator and programmers.

In the past the GSDB biology staff received nightly reports of new gene names, new product names and new miscellaneous features that were added to the database. These reports were reviewed and corrections were made to the data when necessary. For example the misspelled product name "portein kinase C" would appear in the report and would be corrected to "protein kinase C". We anticipate reimplementing the gene name, product name, and taxonomy data reports to help 
maintain the quality and consistency in these data fields. GSDB also intends to reimpliment the miscellaneous feature report and a comment report. Since GSDB has added support for several data types that are not supported by GenBank it is important for GSDB to monitor the "catch-all" data fields to identify data that can be better represented in GSDB by one of the new data types. For example, information about sequence alignments frequently appears as comments attached to miscellaneous features in GenBank flatfiles.

\section{Automatic Vector Analysis}

The algorithm to identify vector contamination of sequences within GSDB has been manually operated and reviewed during FY97. One goal of GSDB for FY98 is to convert this manual process to an automated program that examines each sequence as it is deposited in GSDB (regardless of whether the sequence is obtained via direct submission, data mining or importation from the International Collaboration). It is anticipated that the program will provide a nightly report of the sequences that fit a stringently defined set of conditions and that have been automatically processed, a report of suspicious sequences that need manual review and a report of sequences which appeared to be free of vector contamination. We will also make this algorithm available to researchers from our web site so that they can examine sequences of interest for vector contamination prior to deposition in the database.

\section{Expansion of GSDB and Five year plan:}

Future plans discussed above are already being implemented GSDB function. An additional 5 year plan to expand and add new features to GSDB is outlined below.

\section{Situation Analysis:}

- GSDB is in the information technology business, primarily in the "functional genomics" arena. 
- Our customer base is all individuals involved in molecular biology, genetics and genomics. These entities are the Biotech, Pharmaceutical, Patent attorneys, Educational institutions and the US and State Governments.

- We provide an information system that allows the customers to query the data and ask questions of the data not available elsewhere. We also provide unique data sets that do not appear in other public databases or the international consortium of databases (GenBank, EMBL or DDBJ).

- Generally we are perceived as very user responsive, and friendly. Many of our customers prefer to work with GSDB as opposed to GenBank which is generally seen as unresponsive to the community.

- Customer satisfaction is fair, to poor, generally because the data is difficult to view since the only current way to view the data is through a IC flatfile or via Annotator. Annotator is difficult to use and requires a large investment in computer memory and speed, very few customers have a workstation of this nature.

- Additional products requested by our customers include: GSDB flat files extensions, Web views and the ability to do homology searches (BLAST) against GSDB. Longer term projects include "Functional Genomics", this catch all term includes the ability to query mapping information, expression data, proteins, biochemical pathways plus cell processes.

- The current perceived competition comes from NCBI GenBank, although we are not trying to compete directly, the nature of GSDB and GenBank promotes this attitude. Additional competition can come from other private companies such as Incyte since they offer curated public data plus proprietary data.

- Major trends are in the fields of "functional genomics" in particular expression data, biochemical pathways, and biodiversity.

- Our strength is in the area of bioinformatics; this includes programming, web design and functional biology. Our current weaknesses is in the delivery of necessary tools in a timely manner. Also the perception is that our data needs extensive QA/QC. Furthermore, concerns have been expressed that programming code going to $\mathrm{MII}$ might jeopardize our status as an independent and objective provider of data. 


\section{Operational Strategy}

- Year 1 GSDB will initially concentrate on customers interested in Human and Microbial Genomics. The primary focus in year one will be in completing the Human chromosome discontiguous sequences maps. This task is currently scheduled for completion by Aug. 1 1997. Additional emphasis will be on microbial genomes. We will initially set up homology services, biochemical pathways, expression data sets and data mining for microbes. By collaborating with individuals who have microbial genomic data that is NOT available from the IC databases, we will produce unique data sets.

- Year 2 will concentrate on refining year 1 data tools. Agricultural genomics will become a major area of expertise. Additionally, we propose to collaborate with Genome Therapeutics on their TB and Leprosy data sets. Mitochondria data sets which demonstrate newly discovered information concerning neurological diseases will be added to GSDB. We are discussing possible collaborations with DARPA on emerging infectious diseases or other related projects of interest to DARPA such as algorithms to predict resistance due to genetic changes.

- Year 3 will focus on the development of diabetes /clinical information. This project will expand the existing years projects by adding disease specific biochemical data to the pathway data sets, biodiversity of human populations in relation to type 2 late onset diabetes and other population biodiversity from UNM collaborators.

- Years $\mathbf{4}$ and $\mathbf{5}$ will add completed genome comparison algorithms. Within year four there should be sufficient fungal genomes completed to do genomic comparisons. Lastly, expansion of data sets to incorporate metastasis as a specific data set to GSDB will be undertaken.

\section{Where we will be in Five years:}

- In five years we will be recognized as a world leader in genomic information, particularly in "functional genomics".

- We will be the leader in agricultural genomics and will be the national repository for such information. (not funded by DOE) 
- We will have produced an advanced set of tools that the bioinformatics community will use broadly.

- A virtual laboratory will be available on the web with emphasis on providing training in genomics and molecular biology. (not funded by DOE)

\section{Personnel Resources required for Continuation and expansion of current GSDB functions}

\section{Curation and Annotation of GSDB:}

Tasks: This is an extension of what GSDB is currently doing, except on a much larger scale. It will require an expansion into biological disciplines with skills sets for each area of curation; Human/Mouse, Microbial, Agricultural, and Proteins sections. This is currently the role of the Bioinformatic Associates (MS level biologists) and includes standardizing key words, products, and constructing the discontiguous human chromosome sequences. This function will be reorganized into the above disciplines with both automation and manual biological annotation. It will also include more aggressive data mining and linking with other data sources.

Resources: Currently, this groups includes $2 \mathrm{Ph} . \mathrm{D}$., $5 \mathrm{MS}, 3 \mathrm{BS}$ and 1 post doc level geneticists/molecular biologists (which are counted in the reorganization below).

Reorganization would include Human/Mouse 1 Ph.D., 2 MS level geneticists; Microbiology, 1 Ph.D., 2 MS level prokaryotic and possibly viral geneticists; Environmental. I Ph.D., I MS level. Experience of Post Docs would depend on annotation needs.

\section{Programming services:}

Tasks: Continuation of the software development and programming required to maintain and operate GSDB. It will require a substantial development initiative to create the WEB interfaces to GSDB, these would include a viewer of the data, queries, homology services, integration of other tools necessary for the curation and annotation of GSDB. This task 
component will also develop interactions with other databases through CORBA or other distributed object mechanisms.

Resources: Currently this staff includes 12 personnel made up of 4 Web developers, 6 programmers, 2 DBA's, and 2 system administrators. Additional personnel will include 1 web developer, and 4 programmers.

\section{New GSDB Functions}

\section{Assembly:}

Tasks: Support large-scale sequencing centers that just sequence and do not assemble sequence data. This will allow GSDB to take $3 \mathrm{~kb}$ fragments and assemble them into complete clones and assemble larger contigs from overlapping clones. These large contigs will go to the Oak Ridge National Laboratory's (ORNL) Grand Challenge Project (next section) for automated annotation and then be returned to GSDB. These assembled fragments will not be exchanged with GenBank since it cannot support sequences larger then $350 \mathrm{~kb}$.

Resources: Additional 4 Master's level associates and 1 post doc.

\section{Collaboration with Grand Challenge Project (GCP).}

Task: Data assembled by GSDB from small fragments into large contigs will be shipped off to the GCP at ORNL, this will be either in a GIO file format and/or through the development of a direct database connection between GSDB and ORNL. These larger contigs will be analyzed by the GCP through various algorithms as outlined in the GCP. This annotation will then be added back into GSDB by GIO or direct database connection. This data will not be available to the International Collaboration (IC) databases due to their inability to represent these data. NCGR/GSDB is a component of the Grand Challenge proposal and is already committed to the project. The GCP has just been funded for 3 years at $\$ 12$ million, GSDB will receive $\sim \$ 1$ million from this project over that period. 
Resources: One additional programmer to set up database automation. Once this component is running, few programming resources will be required. Subsequent work will be done by the annotation staff.

\section{Asn.1 Tools:}

Tasks: There is a need to generate Asn. 1 file format for data submitted to GSDB; this would allow the owner of the data to submit data to GenBank without reformatting the data themselves. Several researchers are interested in helping GSDB generate these tools. Resources: 1 additional programmer to develop tools. Once service is established this programmer can move into GIO/Asn. 1 tools development.

\section{Summary:}

NCGR recognizes that the needs of the Genomic Communities are rapidly changing from just archival management of DNA sequence data to a fuller understanding of the biology associated with these data. These new areas of interest include representation of expression data, biochemical pathways, cross species genome comparison, polymorphism, and biodiversity. As a result, this proposed modification of GSDB will move away from it's current mission of direct data submission and curation to concentrate more fully on curation, annotation, data QA/QC, and other aspects of data integration. This proposal broadly outlines the different facets of this modified mission and identifies the resources required for its implementation. 
Table of Current and New Tasks for GSDB.

\begin{tabular}{|c|c|c|c|c|}
\hline Project & Current Tasks & NEW Tasks & $\begin{array}{c}\text { Current } \\
\text { Personnel }\end{array}$ & New Personnel \\
\hline Management & $\begin{array}{l}\text { Management of } \\
\text { GSDB and } \\
\text { NCGR }\end{array}$ & $\begin{array}{c}\text { Implement } 5 \\
\text { year plan }\end{array}$ & 4 & none \\
\hline Curation & $\begin{array}{l}\text { Creating human } \\
\text { chromosome } \\
\text { discontigs, } \\
\text { microbial } \\
\text { genomes and } \\
\text { plant maps }\end{array}$ & $\begin{array}{l}\text { Expand group } \\
\text { into disciplines } \\
\text { be more } \\
\text { aggressive with } \\
\text { data mining and } \\
\text { curation }\end{array}$ & $\begin{array}{l}2 \mathrm{Ph} . \mathrm{D} \\
1 \text { post doc } \\
5 \mathrm{MS} \\
3 \mathrm{BS}\end{array}$ & $\begin{array}{l}2 \text { Ph.D. } \\
\text { I MS } \\
1 \text { post doc }\end{array}$ \\
\hline Programming & $\begin{array}{l}\text { Maintenance of } \\
\text { GSDB }\end{array}$ & $\begin{array}{l}\text { Expanding } \\
\text { current services } \\
\text { GIO, Asn.1 } \\
\text { tools, Viewer } \\
\end{array}$ & $\begin{array}{l}12 \text { programmers, } \\
\text { SA's, Web } \\
\text { developers, DBA's }\end{array}$ & $\begin{array}{c}5 \text { additional } \\
\text { programming staff }\end{array}$ \\
\hline Assembly & $\begin{array}{l}\text { Support centers } \\
\text { Process } \\
\text { sequences } \\
\text { Review output }\end{array}$ & $\begin{array}{l}\text { Generate } \\
\text { contigs }\end{array}$ & None & $\begin{array}{l}3 \text { MS Assoc. } \\
1 \text { post doc }\end{array}$ \\
\hline $\begin{array}{c}\text { Grand } \\
\text { Challenge } \\
\text { Project } \\
\end{array}$ & None & $\begin{array}{l}\text { Submission to } \\
\text { and from GCP/ } \\
\text { ORNL }\end{array}$ & None & $\begin{array}{c}\text { Counted in } \\
\text { programming staff }\end{array}$ \\
\hline Asn. 1 & None & $\begin{array}{l}\text { Tools to } \\
\text { generate and } \\
\text { convert } \\
\text { GenBank Asn.1 } \\
\text { data } \\
\end{array}$ & None & $\begin{array}{c}\text { Counted in } \\
\text { programming staff }\end{array}$ \\
\hline & & Totals & 27 & 13 \\
\hline
\end{tabular}

Detailed Budget page is included in beginning of proposal. 
Figure 1. Proportion of sequence accession numbers in GSDB in various taxonomic groups. Plants includes plants and fungi, microbes includes all eubacteria and archaea, but not the microscopic fungi, and rodent + primate includes all non-human primate and rodent sequences.

Figure 2. Proportion of total basepairs in GSDB in different taxonomic groups. Plants includes plants and fungi, microbes includes all eubacteria and archaea, but not the microscopic fungi, and rodent + primate includes all non-human primate and rodent sequences. 